\section{REPENSANDO LOS ESTUDIOS DE SISTEMAS DE INNOVACIÓN. EL SISTEMA CATALÁN DE INNOVACIÓN COMO CASO ESTRATÉGICO DE INVESTIGACIÓN'}

\author{
Manuel Ahedo \\ Universidad del País Vasco \\ Grupo de Investigación "Análisis Social y Organizativo" \\ y Grupo de la Universidad Rovira i Virgili
}

\section{RETHINKING THE INNOVATION SYSTEMS STUDIES; THE CATALAN SYSTEM OF INNOVATION AS STRATEGIC RESEARCH CASE}

\begin{abstract}
The system of innovation or innovation system approach has brought a profound change and improvement in the understanding of the innovation process, mainly by taking into account the national, regional, sectoral and technological dimensions and factors. However, despite its clear knowledge accumulation, it still has analytical limitations with regard to old questions and also to emerging issues. This article looks at the Catalan innovation system, as a strategic research case, in order to point out which are these questions and issues, and to propose some possible ways to advance in the analytical solution, and in general in moving towards a better understanding of the innovation processes in the globalizing capitalism of the $21^{\text {st }}$ century society and economy.
\end{abstract}

KEY WORDS: Innovation system; social sciences' performativity; institutional-cultural approach.

\section{INTRODUCCIÓN}

En las últimas décadas, el estudio social de la innovación con mayor o menor carácter tecnológico desde varias disciplinas de las ciencias sociales ha recibido un creciente interés social, una mayor nivel de sofisticación analítica y una mayor relevancia para las políticas públicas. Dentro de estos estudios, el enfoque de sistema de innovación se ha centrado en analizar la importancia de los factores institucionales en las dinámicas de innovación, especialmente a los niveles global, nacional, regional y sectorial. Se ha reconocido así que la dinámica sistémica e institucional de la innovación es importante para el desarrollo socio-económico de las sociedades, pero todavía falta precisar en qué medida y mediante qué mecanismos es importante, y cómo interactúa con los otros sistemas y factores sociales, culturales e
RESUMEN: El enfoque de sistema de innovación ha generado un profundo cambio y mejora en el análisis de los procesos de innovación, al tener en cuenta los contextos nacionales, regionales, sectoriales y tecnológicos. Sin embargo, tras varias decadas de acumulación de conocimiento, el enfoque de sistemas de innovación presenta todavía una serie de limitaciones en la comprensión de algunas cuestiones tanto tradicionales como nuevas o emergentes. Este artículo explora el sistema catalán de innovación, considerado un caso estratégico de investigación, para indicar cuáles son esas cuestiones y retos, y proponer algunas vías posibles para el avance analítico y un mejor conocimiento de los procesos de innovación en el emergente capitalismo global de la sociedad y economía del siglo XXI.

PALABRAS CLAVE: Sistema de innovación; performatividad de las ciencias sociales; enfoque institucional-cultural.

institucionales. En este sentido, dentro de las importantes transformaciones de las economías y sociedades actuales, se postula que el enfoque de sistema de innovación necesita de una mayor integración analítica y de una visión más holística y reflexiva de la relación sociedad-economía. Esta reflexión y propuesta analítica toma inspiración en el intento de entendimiento y explicación del caso del sistema catalán de innovación. El sistema catalán de innovación, o de $I+D+i$, presenta una alta heterogeneidad y complejidad, junto a una dinámica emergente de formalización e institucionalización, lo cual le hace ser un caso lo suficientemente interesante y sugerente para testar los diferentes enfoques, y proponer nuevas perspectivas.

El conocimiento sobre el sistema de innovación catalán ha alcanzado un importante nivel de entendimiento y descrip- 
ción del mismo (Buson, 2006). Sin embargo, la literatura sobre los sistemas regionales de innovación, de creciente orientación comparada, presenta un alto grado de ambigüedad y opacidad explicativa sobre el caso catalán (Cooke et al., 1998, 2004; Buesa et al., 2006). Algunas caracteristicas significativas del sistema catalán de innovación son un modesto nivel de intensidad en $I+D$, una eficiencia relativa en la relación inputs-outputs, el activo papel de las Universidades en la transferencia de conocimiento para la innovación, y de forma general la importancia del entorno productivo y educativo en la circulación del conocimiento. Sin embargo, para poder capturar toda la complejidad y heterogeneidad del sistema catalán de innovación se considera necesario el uso de nuevas herramientas y aproximaciones.

Tomando inspiración en el sistema catalán de innovación, el artículo propone avanzar en el enfoque de sistemas de innovación en tres direcciones: a) hacia una perspectiva más amplia e inter-disciplinar en torno al concepto de estudios sociales de la innovación; b) hacia una mayor integración analítica de los dos tipos sistémicos de innovación propuestos principalmente por la escuela IKE-Aalborg, el Science-Technology-Innovation (STI) y el DoingUsing-Learning (DUI), en un tipo integrado de Interactive Learning-Innovation (ILI), que pivota principalmente sobre los mecanismos de interacción formal e informal entre los actores principales del sistema; y c) metodológicamente, hacia un mayor aprovechamiento de las ventajas de estrategias metodológicas de análisis comparados, basados en problemas y preguntas relevantes, y con un importante papel para la metodología cualitativa evolutiva. Con esta estrategia, ilustrada con ejemplos empíricos de una investigación en curso sobre el caso, se aspira a obtener un mayor nivel de conocimiento sobre el sistema catalán de innovación, y a avanzar los estudios sociales de la innovación y del enfoque de sistema de innovación.

La economía catalana vive horas bajas en su historia. Durante los años 50 y 60 su industria le daba uno de los mayores PIB per cápita de España, y durante los años 70 y 80 , el crecimiento económico se basó fuertemente en la inversión de capital extranjero, pero en las últimas dos décadas el crecimiento ha sido menor y con bases de mayor riesgo. Ha descendido sustancialmente su porcentaje de producción industrial, que sin contar la construcción es actualmente el 17-18\% del VAB, y ha crecido fuertemente el sector de servicios, que en un alto grado han sido de poco valor añadido y vinculados al turismo, ocio, comercio, etc., atrayendo un alto porcentaje de mano de obra extranjera. La sociedad catalana es muy diferente a la de hace 150 20 años, y su economía y sus empresas han perdido peso y competitividad. ¿Debería y podría Cataluña encontrar en la investigación y en la innovación unas mejores bases de crecimiento, prosperidad y bienestar? Una parte importante de la ciencia económica y social parece indicar que sí. Sin embargo, aunque los indicadores macroeconómicos de competitividad y de la situación de la I+D en Cataluña sean superiores al tradicionalmente bajo promedio español, tampoco permiten afirmar que el sistema de $I+D$ catalán esté firme en el camino hacia la nueva competitividad y prosperidad basada en la innovación.

Desde el 2006 el gobierno catalán, la Generalitat, ha publicado: a) informes periódicos sobre la situación de la innovación en Cataluña en el 2006 y 2009, con un mayor esfuerzo analítico; y b) informes anuales sobre la I+D y la innovación en Cataluña (2008, 2009 y 2011), de carácter altamente descriptivo ${ }^{2}$. Estos informes han contribuido a la observación y debate sobre la evolución del sistema de innovación para poder tener una visión que inspire las decisiones políticas. Algunos de estos informes han sido elaborados por investigadores académicos, como el primer informe (Buson, 2006) realizado en colaboración con el Colegio de Economistas de Catalunya, el cual ha marcado algunas pautas básicas seguidas en el resto de los informes. Con un fuerte tratamiento comparado, tanto a nivel de España como europeo, la imagen principal que transmiten estos informes es que Cataluña avanza poco en sus gastos de $I+D$, y que todavía le queda mucho para mejorar su sistema, especialmente en lo que se refiere al gasto empresarial en I+D. Estos informes son un buen punto de partida para avanzar en el estudio y comprensión de la innovación en Cataluña, para profundizar y ampliar la reflexión y debate sobre la economía catalana, y sobre las políticas públicas de innovación, junto a las de investigación, industriales, de competitividad, educación, etc.

Este artículo pretende echar un poco de luz sobre el funcionamiento del sistema catalán de innovación y sobre el papel y el efecto que ejerce la interpretación del sistema en la formulación de políticas públicas para la innovación y la competitividad en general. En primer lugar se discute el limitado alcance de los análisis existentes sobre los sis- 
temas regionales de innovación (SRI). En segundo lugar se propone avanzar hacia una visión más amplia de la innovación a través de los conceptos de colaboración, aprendizaje y experimentación. En tercer lugar se describen los rasgos que hacen del SRI catalán un caso altamente complejo para ser analizado con las herramientas convencionales. En cuarto lugar se presenta una propuesta metodológica para el análisis del sistema catalán de innovación, a través de una estrategia de comparación estratégica, que se ha experimentado en un proyecto comparado sobre el papel de las PYMEs industriales en los SRIs de las tres Comunidades Autónomas más industriales y tecnológicamente avanzadas de España (Cataluña, Euskadi y Navarra). Finalmente, se presentan las conclusiones.

\section{El alcance limitado de los anÁlisis DE SISTEMAS REGIONALES DE INNOVACIÓN (SRI)}

La contribución más importante de la perspectiva SRI, dentro de la literatura de sistemas (nacionales) de innovación, ha sido el reconocimiento de la importancia de la proximidad geográfica para la generación, circulación, distribución y aplicación del conocimiento (Olazaran y Gómez-Uranga, 2001; Olazaran y Otero, 2009). El reconocimiento del conocimiento tácito, no-codificado e incrustado en las prácticas sociales da la razón al argumento de la proximidad. Esta dimensión enfatiza la importancia de las relaciones sociales, y de la interacción más o menos organizada, para la circulación y aplicación del conocimiento. El único problema es que la proximidad puede presentar limitaciones respecto a la masa crítica necesaria para la generación de nuevo conocimiento.

\subsection{Realidad, interpretación y acción política: profecías que se auto-cumplen y el caso del SRI normativo}

En la constitución o construcción de la sociedad actual, la importancia de la relación conocimiento-realidad ha recibido diversos tratamientos: la doble hermenéutica (Giddens), la relación entre doxa y habitus (Bourdieu), la sociedad como una distribución general de una profecía que se auto-cumple a través de prácticas (Barnes). Law y Urry (2004) avanzan la discusión sobre cómo la ciencia social contribuye a crear la propia realidad social. Cada vez hay menos líneas claras entre, por una parte, el análisis de la realidad $y$, por la otra, el conocimiento derivado de la interpretación de la misma, la cual tiene una dimensión normativa, en su relación a priori o a posteriori, directa o indirecta con la actuación política y las políticas públicas. Las ciencias sociales en la actual sociedad del conocimiento no pueden ser ajenas a su papel de autoridad epistémica y al uso aplicado de su conocimiento por las instancias públicas, y a su poder de moldeado de la propia realidad social. Dentro de los diferentes tipos de profecías que se auto-cumplen (PAC), Biggs (2010) incluye el más polémico: el de la teoría social, en el que una teoría científicosocial indica cuál es el modelo normativo más adecuado de sociedad, donde los actores involucrados, creyendo en la teoría, construyen la sociedad que acaba asemejándose a la teoría ${ }^{3}$.

El enfoque de los SRI, así como el de clusters, se ha convertido en uno de los productos estrella de la industria del conocimiento del desarrollo regional, especialmente en Europa, y por lo tanto ha inspirado un buen número de actuaciones públicas. Naranja et al. (2008) y Uyarra y Flanagan (2009) analizan el proceso cognitivo en las políticas de innovación a nivel regional, y discuten el papel de la visión normativa de SRI, así como del concepto clusters, y argumentan que ambos conceptos, por su naturaleza ambigua y flexible, han sido una importante inspiración de diferentes tipos de políticas públicas estatales, regionales y locales. Navarro (2009), respecto al enfoque SRI, recoge la diferenciación entre sistema conceptual y sistema operacional, y la emergencia del enfoque normativo de SRI. Navarro retoma la crítica de Cooke a la corriente de los sistemas nacionales de innovación (SNI), la de haber tendido excesivamente al estudio de sistemas operacionales y pre-normativos, sin haber desarrollado previamente el sistema conceptual. En este sentido se puede argüir que las políticas no hacen los sistemas, pero las políticas públicas sí ejercen un papel importante en la constitución y evolución del sistema. Para poder medir los efectos de las políticas públicas sobre los sistemas es necesario tener en cuenta dos cuestiones.

En primer lugar, el proceso de aplicación de la teoría científica normativa, en el que los actores y la lógica del sistema ejercen una fuerte capacidad de influencia para integrar el concepto aplicado a la lógica e intereses del sistema. Por ejemplo, en el caso de las políticas de cluster de Euskadi y 
de Cataluña, aunque partieron de un misma receta cientifica normativa, inspirada en los trabajos del economista M. Porter, el proceso de adopción y aplicación fue muy diferente, debido a que el sistema empresarial, industrial y productivo, y sus actores más relevantes, influyeron de forma significativa con sus particulares estrategias e intereses (Ahedo, 2006).

En segundo lugar, la importancia de la estabilidad y continuidad temporal de una política pública industrial, de innovación, etc., ya que con el tiempo esta actuación política y las prácticas que genera se pueden institucionalizar y así entrar a formar parte de las lógicas del sistema. Por lo general, en nuestra sociedad del conocimiento, a las políticas públicas se las somete de forma creciente a evaluaciones de los resultados, una vez transcurrido un periodo de tiempo, más corto-medio que medio-largo. Este tipo de evaluaciones, a veces realizadas por empresas privadas, suelen jugar al equilibrio positivo-critico, generando así una presión empática hacia la adopción de nuevas recetas o políticas, en las que en muchos casos las propias empresas privadas tienen un interés partidista. La falta de la necesaria continuidad de las políticas públicas durante un tiempo coherente a los resultados esperados se ha convertido en un problema de las sociedades actuales ${ }^{4}$.

\subsection{Un meta-análisis de la visión estrecha (STI) y la visión amplia (DUI) del sistema de innovación en los países nórdicos}

En la literatura, especialmente al nivel europeo, ha empezado a consolidarse la existencia de dos visiones del sistema de innovación: el modelo STI (Science-TechnologyInnovation), y el modelo DUI (Doing-Using-Interacting). El modelo STI ha sido el modelo convencional del estudio linear de los procesos de innovación desde la ciencia y la tecnología a la innovación y aplicación. Esta opción ha tenido inspiración en la literatura y realidad de países anglosajones, y especialmente los Estados Unidos de América, en donde la Ley Bayh-Dole de 1980 formalizaba la capacidad de las universidades para comercializar su investigación, una actividad ya pre-existente en muchas universidades estadounidenses (Smith, 2008). Por su parte, el sistema DUI ha surgido del énfasis en la interacción formal e informal entre múltiples actores, especialmente en el sistema productivo, de mercado de trabajo y de formación y aprendizaje. La distinción entre los dos tipos ha venido en gran parte desarrollada en una parte de la literatura nórdica sobre el tema. Mientras autores suecos, como Edquist (1997) o Fagerberg (2009), han priorizado el enfoque STI, algunos autores daneses, liderados por el grupo IKE-Aalborg (Lundvall, 1992), han enfatizado la importancia de la dimensión DUI, a veces también llamada la visión amplia del sistema de innovación. Lundvall et al. (2002) recogen el surgimiento y la evolución de este enfoque amplio, inspirado en la realidad danesa, y de su posible aplicación al estudio de las economías en desarroIlo. En suma, todo indica que Suecia y Finlandia parecen haber inspirado empírica y normativamente el modelo STI, mientras Dinamarca ha inspirado el modelo DUI.

¿Por qué los países nórdicos han constituido una especie de laboratorio para el análisis de los sistemas de innovación? Estos países presentan una serie de características que les hace buenos candidatos al trabajo de investigación y experimentación social, entre las que se pueden destacar: su histórico alto nivel educativo, su reducido tamaño, sus efectivos mercados laborales, sus economías abiertas al comercio internacional, la existencia de un porcentaje importante de PYMEs junto a grandes empresas, y finalmente por sus prácticas de monitorización mutua y de imitación institucional, todo ello coordinado por un Estado altamente interventor, mayormente a través de diferentes políticas de bienestar, sociales y educativas, y de forma más modesta en lo que se refiere a las políticas industriales y de innovación. Sobre estas premisas, se pueden plantear dos cuestiones:

¿Por qué el modelo STI se fija crecientemente en Suecia y Finlandia? Suecia y Finlandia suelen encabezar los rankings europeos sobre inversión en innovación, especialmente en I+D formal y en educación e investigación superior. Según Eurostat, del porcentaje de PIB invertido en I+D, en educación superior, en 1998 Suecia y Finlandia invertían el 0,80 del 3,60 y el 0,56 del 2.88 , y en 2008 , el 0,80 de 3,75 , y el 0,70 del 3,80 , respectivamente. En Finlandia el gasto del gobierno en la I+D ha sido también alto: el 0,36 en 1998 (el 0,12 en Suecia) y el 0,30 en el 2008 (el 0,17 en Suecia). Al coincidir estos gastos e in-puts con datos macroeconómicos no negativos, la prueba de la eficacia de la fuerte inversión en I+D queda probada. Suecia era ya un pais de referencia durante los años 70 y 80 , y tras la crisis de los 90, en los últimos años ha vuelto a la senda del crecimiento. Finlandia sufrió una fuerte crisis en los años 80 y primera parte de los 90, para, a continuación, apostar institucionalmente por la estrategia de la sociedad 
de la información y del conocimiento, como medio para salir de la crisis, con resultados positivos reflejados en sus resultados en los estudios PISA, y en el éxito de empresas emblemáticas como Nokia, a pesar de los negativos datos macroeconómicos más recientes.

¿Por qué el modelo DUI es propuesto por el grupo IKE de Dinamarca? En Dinamarca, la inversión en I+D es tradicionalmente más baja que en Suecia y Finlandia, y se ha concentrado en los últimos años en la educación superior, tal y como se puede ver en los porcentajes de PIB invertidos en I+D: en 1998 era el 0,41 del 2,04, y en el 2008 era el 0,71 del 2,72 (Eurostat). El grupo IKE de la universidad de Aalborg, liderado por Lundvall, supo ver que en la economía y sociedad danesa los elementos y factores de su competitividad se fundamentaban más en el sistema empresarial (de relaciones entre empresas y entre clienteproveedores) y en el sistema laboral y de organización del trabajo. Llama la atención que el "descubrimiento" de la flexiseguridad del mercado laboral tuvo también lugar en la Universidad de Aalborg, una universidad pequeña, joven e innovadora de la periferia danesa en el Norte de Jutlandia. Datos recientes sobre los últimos años indican la existencia de problemas en la competitividad y en el mercado de trabajo danés, lo cual se puede relacionar con quizá un mal uso de la auto-interpretación institucional del éxito de su flexiseguridad a la hora de tomar decisiones públicas. En una perspectiva meta-analítica, las contribuciones científicas de los grupos de investigación de la universidad de Aalborg, inspiradas en el análisis de su propio país desde una visión directa o indirectamente comparada, han contribuido de forma positiva al desarrollo teórico, analítico y normativo de los sistemas de innovación.

La mejor lección que se puede obtener de estos paises nórdicos es su avance hacia la integración de los dos subsistemas o tipos, el STI y el DUI, ya que la existencia efectiva y complementaria de ambos es la mejor garantía para hacer frente a los retos económicos y sociales actuales. Según Asheim (2009), Finlandia es quizá donde se están dando los mayores avances en esta dirección.

\subsection{Tipologías de SRls: función descriptiva y débil análisis}

Una de las tareas de las ciencias sociales es la categorización y la tipologización de las realidades sociales. En la ciencia social comparada, la realización de taxonomías es una tarea quasi-natural. Pero, ¿cómo contribuye la tipologización al conocimiento, análisis y explicación de los casos particulares? En la literatura sobre SRIs han aflorado muchas tipologías.

Cooke et al. $(1998,2002)$ proponen 9 tipos diferentes de SRI, según las combinaciones de dos variables: la forma principal de innovación por las empresas (globalized, interactive y localist) y la gobernanza del apoyo a la innovación en las empresas (grasroots, network y dirigiste). Cooke (2006) integra varias propuestas en tres grandes tipos:

a) el "locally embedded" o "grasroots RIS": un SRI incrustado territorialmente, en el que las empresas (usando conocimiento sintético) basan su innovación en los procesos de aprendizaje inter-empresarial y localizado, estimulado, pero la conjunción de la proximidad relacional geográfica sin mucha interacción con las organizaciones generadoras de conocimiento (universidades y centros de I+D). Los distritos industriales italianos son uno de los ejemplos de este tipo de SRI. Este tipo de SRI recoge asimismo el espíritu de la definición amplia de sistema de innovación;

b) el "regionally networked" o "network RIS": este modelo sería el tipo más ideal, y se caracteriza por la existencia de cluster(s) regionales rodeados de una infraestructura de instituciones de apoyo, dentro de una mayor cooperación público-privada, y un mayor papel regional de los centros de I+D, de las organizaciones de formación y de otros apoyos a la innovación. Clusters industriales regionales de tecnologías medias y altas suelen predominar en este tipo de SRI;

c) "regionalized national" o "dirigiste RIS", que tiende a operar bajo la lógica de STI, impuesta desde las instancias centrales de las políticas científicas y tecnológicas, como son los casos de los parques científicos de Francia, o los clusters estratégicos promovidos por el Estado noruego.

El caso catalán en esta literatura tiende a aparecer incluido en el tipo de "locally embedded grasroots", aunque cuando se trata de la innovación en sectores emergentes de la ciudad de Barcelona se incluye a veces en la categoria de "networked". Las tipologías de SRIs nos ofrecen información de la diversidad de soluciones y funciona-

ARBOR Vol. 188753 enero-febrero [2012] 49-62 ISSN: 0210-1963 
mientos sub-nacionales al reto de la economía de la innovación, y por lo tanto permite relativizar los posibles análisis normativos basados en tipos ideales teóricos. Pero, además de esta función, ¿ayuda esta tipologización a conocer los aspectos positivos y negativos del SRI catalán, sus problemas sistémicos y sus posibles soluciones de mejora? Hay claras dudas de que ese sea el caso, tal y como se puede deducir de la ambivalencia de los informes anuales institucionales y de los problemas de la literatura secundaria sobre el caso para superar los discursos oficiales y más institucionales sobre el tema. Se considera, por el contrario, necesario desarrollar metodologías de estudios de caso longitudinales en profundidad cuantitativa y cualitativa, dentro de una comparación indirecta con adecuadas referencias de contraste. Se postula que lo idóneo es que el análisis comparado entre casos debe ir en paralelo a estudios de caso en profundidad, en una relación de complementariedad y fertilización mutua.

\section{LOS ESTUDIOS SOCIALES DE LA INNOVACIÓN: HACIA UNA VISIÓN AMPLIA Y MULTIDISCIPLINAR}

En esta sección se apuntan los caminos por los que el estudio de la innovación, y en concreto el enfoque RIS, puede mejorar y desarrollarse. Estas indicaciones se sitúan dentro de una perspectiva (neo)-institucional, con un énfasis en los elementos sociales y culturales.

\subsection{La escondida innovación al nivel subnacional: instituciones, sectores y empresas}

El desarrollo, circulación y aplicación de conocimiento tiene lugar en las relaciones entre agentes y organizaciones en un contexto institucional. El contexto institucional establece las regulaciones, las normas y convenciones, y los mapas cognitivos o de creencias, que estimulan la relacionabilidad, e influyen en la forma y el contenido de las relaciones y de la interacción entre actores. Mientras las instituciones regulativas operan al nivel de estado-nación, las normativas y especialmente las cognitivas operan al nivel de mayor relación directa o mediada entre actores. En este sentido, la geografía y el sector productivo o tecnológico o adquieren relevancia. En contextos de proximidad donde hay una alta concentración sectorial, las instituciones estimulan la relacionabilidad entre actores.
El nivel sectorial es una dimensión clave. La estructura sectorial de una economía es el resultado de un proceso histórico y de construcciones y (re)producciones constantes. El sector de actividad, visto como campo interorganizativo, en la sociología institucional, muestra importantes dinámicas autónomas en nuestras sociedades de avanzada diferenciación interna. La economías actuales, además de los tres sectores clásicos, han viso aparecer nuevos sectores de actividad cuaternarios y quinarios, como una forma de clarificar la gran categoría del sector servicios. El subsector de servicios de valor añadido a las empresas es considerado de especial importancia en las economías desarrolladas. Dentro del sector industrial, los sub-sectores de actividad pueden variar de actividades de alto, medio y bajo nivel de conocimiento, y entre sectores de productos finales y de productos intermedios. Todas estas variaciones sectoriales son de vital importancia.

Finalmente, las empresas, tanto las grandes como las PYMEs, juegan un papel clave en el sistema de innovación, a través de la organización del trabajo, la relación entre el mercado interno y externo de trabajo, la formación continua de los empleados, etc. Es por lo tanto necesario prestar una mayor atención a la realidad empresarial en el análisis de los sistemas de innovación. En este sentido, sería necesaria una mayor fertilización entre los enfoques de sistemas de innovación con los sistemas empresariales (Whitley, 1999, 2002).

\subsection{Hacia el modelo integrado STI-DUI}

Si la inclusión del modelo DUI supone la ampliación del tradicional modelo STI, el siguiente paso es la búsqueda del equilibrio efectivo entre la dimensión STI y la Interactive-Learning-Innovation en todo sistema de innovación nacional o regional. Efectividad queda cercana a eficacia y eficiencia. Sobre la eficiencia de la innovación, estudios estadísticos sistemáticos recientes ofrecen material interesante para la reflexión. En la European Community Innovation Survey (2004) España, en lo que se refiere a los inputs, se incluye dentro del grupo de moderate innovators junto a Chipre, la República Checa, Estonia, Italia, Noruega y Eslovenia, pero en términos de eficiencia España mejora su posición en el ranking. El estudio estadístico de Hollanders y Esser (2007) indica que los países del grupo de moderate innovators presentan "a range of different efficiencies combining all possible combinations of above 
or below average efficiency performance" ${ }^{n}$. En este análisis España aparece por encima de la media de eficiencia en propiedad intelectual, y en lo relativo a los beneficios económicos o ventas derivadas de productos mejorados 0 de procesos. Los paises nórdicos presentan niveles moderados de eficiencia, especialmente, Suecia. En esa línea, en el estudio estadístico Arundel et al. (2008), enfatiza la importancia de los innovadores rechazados (neglected innovators), es decir, las empresas que innovan pero que no hacen I+D formal, y recuerda la literatura que en los últimos años ha resaltado la importancia de múltiples formas de innovar ${ }^{6}$. Indican que si la media de la UE-25 es de $50 \%$ de empresas innovadoras $\sin I+D$, hay paises que presentan un porcentaje mucho mayor: Bulgaria (79\%), Islandia (69\%), Rumanía (65\%), y España (60\%), mientras países como Noruega, Bélgica y Grecia están por debajo del $41 \%$. La relevancia para las políticas públicas de este tipo de estudios es evidente ${ }^{7}$. Estos dos tipos de estudios ponen en cuestión los tradicionales enfoques del sistema de innovación, dominado por una visión STI y formal.

\subsection{La sociologización de la sociedad del aprendizaje y la innovación}

El desarrollo de los estudios sociales de la innovación se ha caracterizado por un enfoque micro y de proceso, inspirados en los estudios sociales de la tecnología, que ha servido para cuestionar la tradicional explicación linear de la ciencia económica (Callon, 2007). Sin embargo, en la sociedad actual, dominada por el papel de la información y del conocimiento en la era de la globalización, la innovación tecnológica y organizativa se ha convertido en parte constitutiva de la sociedad en sí, y son necesarios enfoques más interdisciplinares que integren la dimensión micro, con la más macro o institucional, sin perder la atención a la evolución y los procesos (Casper y Van Waarden, 2005).

Los estudios de los SNI y SRI han sido mayormente realizados por investigadores situados en la economía evo-

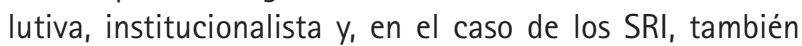
en la economía regional y la geografía de la economía. La contribución de las otras disciplinas de las ciencias sociales ha sido importante, pero no han podido alterar las prácticas científicas de la ciencia económica, con lo que no se ha contado con una parte importante en el estudio de la evolución de las relaciones sociales respecto a la innovación, en contextos más o menos organizados e institucionalizados. Un ejemplo es la cuestión de los bienes colectivos, que la ciencia económica tiende a ver como bienes de club o de grupo, y por lo tanto más privados que públicos, o la colaboración público-privada, de la que la ciencia económica tiene dificultades para operacionalizar sus supuestos micro-conductuales individuales. Otro ejemplo son las variables organizativas e institucionales que influyen en las relaciones entre actores y agentes. Es necesario el desarrollo de una disciplina más amplia, unos estudios sociales de la innovación, que tengan por objeto de estudio los fenómenos micro o procesuales, como los macro o sistémicos. Se propone así un enfoque interdisciplinar para el estudio de la relación sociedad-innovacióneconomía.

Este nuevo enfoque podría servir para dar cabida a una mayor contribución de disciplinas como la sociología, y su capacidad para analizar dimensiones sociales y culturales. Por ejemplo, la sociología podría identificar las formas de relación social que se correlacionan con los diferentes tipos de base de conocimiento operacionalizadas en la innovación. Asheim y Coenen (2007) proponen tres tipos de conocimiento según el tipo de industria y actividad sectorial: sintético, analítico y simbólico, en el que las formas de relación de rumoreo y de cara a cara tienen diferentes niveles de importancia. En las industrias de base de conocimiento sintético (de ingeniería o manufactura), que suelen estar concentradas espacialmente, el cara a cara es importante para la transmisión de conocimiento tácito en relaciones verticales (productor-usuario o cliente y subcontratista); este tipo de conocimiento es más circulable en formas sociales de tipo comunitario. En las industrias de base de conocimiento analítico (o científico), características de sectores de alta tecnología, la relación cara a cara es altamente apreciada en relaciones horizontales entre investigadores no limitadas al plano local; este tipo de conocimiento es más fluido en formas sociales asociativas. Finalmente, en industrias creativas que descansan en una base de conocimiento simbólico y creativo, en cambio, tanto el cara a cara como el rumoreo puede ser importante como vía de intercambiar conocimiento, y la proximidad resulta necesaria a través de formas sociales de tipo red informal, o coyunturales en torno a proyectos. Por lo tanto, podemos tener tres tipos de conocimiento, según la industria, que se relacionarian con tres tipos básicos de organización de la interacción: la comunitaria, la asociativa y la informal en red.

ARBOR Vol. 188753 enero-febrero [2012] 49-62 ISSN: 0210-1963 
Otros conceptos, que podrían ser clarificadores para la descripción y la explicación de los sistemas nacionales o regionales de innovación, son la colaboración, el aprendizaje y la experimentación. La colaboración es el resultado del proceso que comienza en la interacción, avanza hacia la coordinación y que puede desarrollarse un poco más hacia la cooperación. La colaboración entre agentes del sistema de innovación es un mecanismo que engarza a los actores e integra el sistema. De hecho, el nivel y contenido de la relación entre los agentes del sistema de I+D se suele considerar como la variable básica para poder decir que estamos ante un sistema o no. La colaboración emerge a través de múltiples formas, en la interacción entre agentes en organizaciones, asociaciones, redes, plataformas, clubs, foros, etc. El aprendizaje es un proceso por el que un actor 0 un sistema tiene acceso a otra realidad, y una vez entendida, introduce ese conocimiento en su sistema de creencias, lo cual le puede llevar a modificar sus prácticas de conducta e interacción. El aprendizaje ha sido utilizado profusamente tanto para caracterizar la mejora de la innovación en una región o localidad (learning region o localizad learning), como para sugerir procesos de cambio y evolución en las políticas públicas (policy learning). Estos procesos de aprendizaje requieren de una serie de condiciones organizativas e institucionales como reflexividad, criticismo, pragmatismo, diversidad y apertura cognitiva.

Finalmente, la experimentación se refiere a un proceso altamente inductivo de "manos a la obra" (bootstrapping) y de prueba y error, en el que los riesgos son deliberados y compartidos entre los participantes. Los experimentos que evolucionan de manera efectiva y exitosa suelen ser fuente importante de innovación institucional, es decir, son copiados por otros actores, o son institucionalizados y generalizados a través de políticas públicas. Un ejemplo puede ser el proceso de difusión de los Centros Tecnológicos (CT) del País Vasco. Nacidos como apuestas colectivas de colaboración por algunas PYMEs industriales, especialmente en la provincia de Guipúzcoa, se difundieron con apoyo institucional por el resto de las provincias vascas, para pasar a conformar el paisaje institucional a través de las políticas de innovación del Gobierno Vasco. Para el gobierno catalán de izquierdas desde el 2004 al 2010 los CT vascos han sido una referencia a seguir, e influyó claramente en sus programas y políticas de creación y promoción de Centros tecnológicos en Cataluña.
En suma, inspirados en una perspectiva (neo)-institucional y comparada, se propone el concepto de estudios sociales de la innovación, con un adecuado énfasis analítico en los aspectos sociales y culturales de los procesos sistémicos de innovación. Veamos cómo podría ser un esbozo de este tipo de enfoque para el caso del sistema catalán de innovación.

\section{LA COMPLEJIDAD DEL CASO CATALÁN: ¿UN MODELO STI-DUI DE ALTA DUALIDAD O DE BAJA INTEGRACIÓN?}

La economía y sociedad catalanas presentan un alto nivel de heterogeneidad y complejidad. A nivel general, el sector primario es todavía importante (especialmente agricultura, con un 2,5\% de la ocupación), el sector industrial ocupa a aproximadamente el $25 \%$ de la población, pero solo genera el $18 \%$ del $V A B$, y los servicios ocupan el $60 \%$ de la ocupación, todavía por debajo de la media de la UE-15 de un 67-68\%, aunque muchos de estos servicios son de poco o medio valor añadido. Solá et al. (2003) analiza las estadísticas del INE y concluyen la importancia del tamaño de la empresa industrial de cara a innovar. Observa que en el País Vasco las empresas de menos de 250 empleados en los sectores de mayor orientación tecnológica invierten más (55\%) que las empresas de más de 250 empleados (45\%). En toda España las PYMEs suponen aproximadamente el $40 \%$ de la inversión total en I+D, pero el porcentaje del $60 \%$ de las grandes empresas se debe al mayor peso de algunas regiones, como Madrid, donde suponen el $80 \%$, y Cataluña, el 60\%.

\subsection{El sistema de innovación catalán: ¿qué tipo de sistema?}

Como se ha indicado antes, Cooke et al. (2004), en el clásico estudio comparado de SRIs en Europa, caracterizan el sistema catalán como un modelo interactivo en su dimensión de innovación empresarial, y basado en "movimientos de base" (interactive grassroots) en su dimensión de la gobernanza del apoyo a la innovación empresarial. En ese libro, el capitulo sobre Cataluña, altamente cualitativo y descriptivo, se centra especialmente en las políticas públicas, y finalmente incluye un apartado sobre las redes industriales, en las que mayormente se describe el 
sistema de organización de los intereses empresariales. En un estudio cuantitativo comparado de casos regionales en España, Buesa, et al. (2006) analizan el papel de cuatro factores para explicar el modo de funcionamiento de los sistemas de $I+D$ de las regiones españolas, y encuentran que los cuatros sistemas más desarrollados tienen un lógica dominante: en Madrid la Administración Pública, en Cataluña el entorno, en el País Vasco las empresas, y en Navarra la universidad. En su estudio estadístico de las patentes es el factor de entorno de innovación y de producción regional el que más explica el mayor número de patentes, en el que Cataluña destaca sobre los otros casos. La presencia de grandes empresas industriales nacionales e internacionales puede tal vez explicar estos datos. Para Buesa et al. (2006), en el sistema de innovación catalán los elementos clave son los relacionados con el ambiente y las infraestructuras de apoyo, como la estructura productiva (el peso de las industrias con tecnologías bajas, medias y altas, el nivel de exportación, el porcentaje de mano de obra cualificada), ayuda a las empresas a innovar (medida en el número de proyectos de innovación del Ministerio), conocimiento acumulado y tamaño de la región. Las variables seleccionadas para el factor de entorno productivo regional son variables descriptivas y sistémicas (estructura industrial, peso exportador, número de proyectos $I+D$, tamaño de la región, etc.), sin agentes u organizaciones, por lo que es difícil identificar a los agentes dominantes del sistema.

\section{2. ¿La relación STI-DUI? Características del sistema empresarial y de investigación catalán}

En lugar de considerar los modelos STI y DUI como dos enfoques diferentes (estrecho y amplio), se propone su integración analítico-conceptual y a la postre una integración operacional. Se explora a continuación si el sistema catalán de innovación presenta indicaciones de avanzar hacia la integración de los dos subsistemas, el STI y el DUI.

El sub-sistema STI. La investigación en Cataluña presenta unos indicadores bastantes positivos. El sistema público de investigación de Cataluña, liderado por las Universidades, presenta unas buenas estadísticas y posicionamiento en los recientes ranking. En el 2008 Cataluña dedica el 1,61\% de su PIB a I+D y representa casi el 1\% de la producción científica mundial (similar al peso que tienen países como
Finlandia y Escocia), es decir, el 2,5\% de la producción científica de la UE-15 y aproximadamente el 25\% de la producción española, siendo el $20 \%$ de la población. Aquí hay que destacar el temprano interés institucional que el sistema universitario suscitó, y que a lo largo de los años 90 generó la creación de la Universitat Pompeu Fabra, y a finales de los 90 generó el inicio de una serie de políticas universitarias y de investigación, lideradas por el economista de la Universitat de Barcelona, Mas-Colell, como máximo responsable, es decir, como Consejero de Universidades de la Generalitat. El estado de salud del modelo STI en Cataluña, siguiendo los datos recogidos en Buson (2006), indica una relativamente importante relación entre universidades y empresas. Para el período 2001-2003, el $7 \%$ de las empresas innovadoras habían cooperado con universidades, mientras la media española era de un 4\%, y la media de la UE era del 9\%, pero la cooperación con Centros Tecnológicos era reconocida por sólo un 2,5\% de las empresas innovadoras, en la media española de un 2,4\%. Sobre la difícil relación entre universidades y empresas Barge-Gila et al. (2009), según la encuesta de innovación del INE para 2003 y 2004, miden el porcentaje de I+D personal empresarial en las relaciones con universidades y con institutos tecnológicos: del total de España Cataluña tiene un nivel alto en su relación con las universidades (21-22\%) aunque las relaciones con los Institutos Tecnológicos parece estar aumentando (del 9\% en el 2003 al $13 \%$ en el 2004).

El sub-sistema DUI. La estructura sectorial industrial incluye una amplio espectro de actividades y productos, que van desde la tecnología y conocimiento de nivel alto (biomedicina, electrónica y TIC, salud, etc.), de nivel medio (química, automoción, mecánica, metal, etc.) hasta baja (mueble, textil, calzado, etc.). El tamaño empresarial es también heterogéneo, aunque predominan las PYMEs, las cuales no suelen invertir en I+D (Buson, 2006). La presencia de capital extranjero en el sector industrial es muy elevada. En Buson (2006) se recogen los datos de la encuesta de innovación del Instituto Nacional de Estadística para el 2003: aproximadamente el 25\% de las empresas catalanas dicen ser innovadoras; el $18 \%$ en proceso, y el $14,5 \%$ en producto. Dentro del sector industrial, el 21\% de las empresas dicen hacer innovación de proceso y el $19 \%$ en producto, y solo el $11 \%$ en ambos. La importancia de las relaciones inter-empresariales y de cliente-proveedor es un poco mayor en Cataluña que en la media española. 
Para el período 2001-2003, en la industria, el 3,7\% de las empresas innovadoras dicen haber cooperado con clientes (el 1,9 de media española), y el $9 \%$ con proveedores (un $6 \%$ de media española). En lo referente a cambios organizativos, los indicadores totales y por sectores de actividad son más bajos que las medias europeas; solo destacan en el apartado de cambios de estructura organizativa total (un $20 \%$ de las empresas y un $11 \%$ en la UE), y en cambios en los conceptos o estrategias de marketing, con un 12,3\% frente a un 5\% en la UE; en el sector industrial el único indicador un poco superior es el de técnicas de gestión avanzadas (con un 14,6\% frente a un 12\% de la UE). En general, el sistema productivo y empresarial, que es el pilar del sistema DUI, da la imagen de relativa baja inversión en I+D o en factores relacionados con la innovación, como la formación, el cambio organizativo, etc.

\section{3. ¿Y las políticas públicas de innovación?}

Estas dos diferentes historias sobre la poca innovación empresarial y la digna productividad investigadora de las universidades y los centros de investigación pueden apuntar a posibles dinámicas de tensión y conflicto entre los intereses y lógicas dominantes de los dos sub-sistemas, en la que de momento parece que los intereses científicos y académicos han prevalecido. Castro et al. (2003) y Sanz y Castro (2005) caracterizan las políticas tecnológicas catalanas como académicas, porque han sido definidas por los colectivos de investigadores de las universidades y destinadas a su interés. De hecho, la Comissió Interdepartamental de Recerca i Innovació Tecnològica (CIRIT) se creó en 1980, antes incluso que la agencia CIDEM que se creó en 1981. El 1. Plan de Investigación es de 1993-1996, mientras que el 1. Plan de Innovación es de 1999-2000 y lo fue a impulso de la iniciativa RITTS de la Unión Europea. En el 2005 se aprueba el 1. Plan de investigación e innovación de Cataluña. En la aplicación y funcionamiento de éste y de los sucesivos Planes integrados de Investigación e Innovación no queda claro el efecto real de impulso de la innovación. En perspectiva comparada, la marcada orientación académica de las políticas tecnológicas de Cataluña contrasta con los modelos más industriales y empresariales del País Vasco o de Galicia.

¿Por qué ha habido fuertes políticas de investigación y pocas de innovación en Cataluña? Según Sanz y Castro (2005), para analizar las opciones de política pública, además de la estructura de intereses, hay también que tener en cuenta otros factores como los presupuestarios, la estructura departamental de los Gobiernos, y la existencia o no de organizaciones que institucionalicen el modelo de políticas. En este último caso cabe destacar el papel del CIRIT (Commisió Inter-Departamental de la Recerca e Innovació Tecnològica), creado a inicios de los 80 y que en los 90 pasó a depender de la Consejeria de Educación y Universidades. La aparición de políticas de innovación más sustantivas y ambiciosas ha coincido con el aumento del discurso y la reflexión sistemática sobre la innovación. El alto foco de atención sobre el sistema STI, y la tradicional comparación con el sistema vasco, ha conllevado que la política de innovación más importante en los últimos años de gobiernos tripartitos desde el 2004 haya sido la creación y promoción de Centros Tecnológicos, y la reorganización del mapa de agentes de transferencia tecnológica, bajo la marca o red TECNIO en el 2008-2009.

\subsection{Hacia un estudio social del sistema de innovación catalán}

Como se ha indicado anteriormente, el mero hecho de seleccionar un tema o un aspecto para el análisis ya implica cierta normatividad latente sobre la importancia del mismo. En este sentido, en el sistema de reflexión oficial sobre el SRI catalán, basado en los informes oficiales sobre la situación de la I+D en Cataluña, el énfasis analítico ha sido mayor en el sistema STI que en el sistema DUI. Asimismo, el análisis ha tendido a ser mayormente descriptivo, en base principalmente a estadísticas descriptivas, que no han dado paso a reflexiones sobre las lógicas del sistema. En la vertiente comparada, se ha tendido a priorizar la comparación con otras regiones europeas, dejando la comparación con otras Comunidades Autónomas españolas relevantes. Sin embargo, no se dispone de ningún estudio de la eficiencia del sistema, ni de las relaciones del sistema con otros sistemas relevantes (empresariales, laborales, etc.) ni de la evolución de la relación entre política-sistema, y de las ideas que inspiran las políticas.

En una perspectiva macro-institucional hay también otros muchos espacios de realidad y dinámicas cuyo análisis bien podría arrojar luz sobre el funcionamiento de la economía y de la sociedad de la innovación y del aprendizaje en Cataluña. Por ejemplo, aunque a nivel institucional quizá haya predominado el subsistema STI, el subsistema DUI ha 
perdurado y mantenido cierta pujanza, para quizá estar conformando un sistema híbrido STI-DUI de baja intensidad e integración. Asimismo, sería interesante observar la relación entre estructura socioeconómica e innovación, ahora que se habla tanto de la importancia del capital social. 0 el papel de las asociaciones profesionales en el sistema de innovación, especialmente de la mano de obra de alta cualificación, y de los vínculos que establecen entre universidades y empresas. Igualmente, analizar las diferencias territoriales en las comarcas más industrializadas podría ilustrar lógicas específicas sobre el aprendizaje localizado (Maskell y Malmberg, 1999) y la relación entre lógicas públicas y privadas ${ }^{8}$.

En suma, se puede argumentar que la interpretación que se ha hecho del sistema de innovación catalán es ambigua, lo que a su vez puede indicar que el sistema catalán no es fácil de caracterizar y entender. Quizás podemos estar ante un modelo particular diferenciado de los propuestos en la literatura, un tipo de SRI que además de presentar una emergente integración de las dos dimensiones STI y DUI, presenta rasgos particulares, como el alto nivel de exportación, una lógica asociativa liberal que estimula un ethos profesional e individualista en el mercado de trabajo y en las empresas, un marcado nivel de variedad territorial local que le da al sistema cierta energía para la necesaria evolución y aprendizaje interno.

\section{Hacia una metodología comparada estratÉgICA}

Por lo tanto, para entender mejor un sistema tan complejo como el catalán se postula la necesidad de observar y analizar el caso con nuevas herramientas y aproximaciones. Para ello se propone una estrategia analítica fundamentada en tres pilares:

a) Análisis comparado (indirecto), en donde los casos de referencia no limitan la necesaria atención al caso a investigar. Los análisis comparados entre casos de RIS no abundan, y cuando lo hacen suelen ser casos de diferentes estados-naciones (Cooke et al., 1998, 2002), lo que conlleva la pérdida de control de variables importantes referentes a los diversos factores institucionales de los estado-nación. Por lo tanto, se propone la conveniencia de comparar casos de RIS dentro de las fronteras estatales, para así poder capturar las particularidades dentro de un marco institucional común. Este tipo de análisis de casos regionales dentro del mismo país tiene la ventaja de permitir una mayor profundización al caso particular en paralelo a los otros casos, una vez que se mantienen estables los factores institucionales comunes. En este tipo de análisis comparado, en los casos aparentemente más difíciles por tamaño u homogeneidad es previsible encontrar relevantes variaciones locales, lo cual podría justificar el estudio de los subsistemas o variaciones locales dentro del caso nacional o regional.

b) Análisis basado en problemas, a través de la identificación de las paradojas, conflictos y contradicciones estructurales y sistémicas. Esto complementaría el análisis directa o indirectamente normativo del enfoque STI, con su énfasis en indicadores convencionales como el nivel de inversión en $I+D$, número de patentes, etc. El estudio comparado de los sistemas de innovación, por cuestiones de comparabilidad y de parsimonia metodológica, suele operar en base a una serie de variables estándar, mayormente cuantitativas. Estos parámetros comparativos vienen definidos dentro de unos enfoques nacidos en contextos de innovación tipo STI, y que por su fuerte presencia en la literatura científica sobre el tema, han ido adquiriendo un mayor estatus y reconocimiento.

c) Metodología cualitativa evolutiva, para observar cómo los agentes y las instancias del sistema interactúan y se relacionan, trayendo dinámicas y fuerzas de cambio y reproducción al sistema. El período adecuado de estudio sería de unas 2 décadas, y en el caso catalán, por la particularidad política española, se debería intentar cubrir desde la década de los 80, es decir, hasta la actualidad una evolución de medio alcance de 3 décadas.

\section{Conclusiones}

En este artículo se ha presentado una revisión crítica de los estudios realizados sobre los sistemas regionales de innovación. En primer lugar, se ha argumentado que las herramientas analíticas desarrolladas no resultan adecuadas para el análisis de un caso tan complejo y heterogéneo como el sistema catalán de innovación. En

ARBOR Vol. 188753 enero-febrero [2012] 49-62 ISSN: 0210-1963




segundo lugar, se han propuesto líneas para su avance y desarrollo, en un movimiento hacia un nuevo enfoque inter-disciplinar de estudios sociales de la innovación, que con contribuciones importantes de la sociología tuvieran por objetivo la exploración de los caminos de integración y complementariedad entre los sub-sistemas o tipos de innovación, el STI y el DUI. En cuarto lugar, se han presentado las complejas características y dinámicas del sistema catalán de innovación, reflejadas en sus débiles políticas de innovación. Finalmente, se propone una metodología estratégica comparativa y evolutiva, adecuada para el estudio y análisis de un caso complejo como el sistema de innovación regional de Cataluña. En suma, el enfoque de sistema regional de innovación sigue siendo una herramienta analítica válida para analizar los procesos de innovación en nuestras sociedades nacionales actuales, pero necesita un proceso de mejora analítica y metodológica.

\section{NOTAS}

1 Este artículo ha sido escrito dentro del proyecto "Pymes Industriales y Sistema Regional de Innovación: el caso de Cataluña" (2099-2011), financiado por el Ministerio de Ciencia e Innovación. Quiero dar las gracias a los miembros del Grupo de Recerca ASO de la URV por su ideas y apreciaciones, y al coordinador de este número monográfico por sus acertadas sugerencias.

2 A través principalmente del CIDEM, la principal Agencia Catalana de Desarrollo del Gobierno Catalán, y desde el 2008 de ACC10 que fusionó el CIDEM con el COPCA, Consorcio para la Promoción y el Comercio Exterior de Cataluña).

3 El ejemplo típico de PAC con un alto nivel de relevancia y sofisticación es el de la burbuja inversora, por el que un número creciente de actores invierten en productos financieros que creen que son rentables, y de los que tienen constancia de sus beneficios, a pesar de estar fundamentados en especulaciones sin base real (el esquema de Ponzi).

4 Por ejemplo, en la Comunidad Autónoma Vasca las actuaciones de politicas de clusters y de Centros Tecnológicos han tenido una continuidad de más de 20 años, y ya se pueden considerar como parte del sistema. El papel público de las asociacionescluster vascas es comparable al estatus de legítimo stakeholder en un sistema de gobernanza corporatista. Por el contrario, en Cataluña, la política de clusters hasta inicios del año 2000 se redujo a un programa de refuerzos al nivel de micro-cluster localizado, llevados a cabo por una empresa de consultoría. Con los nuevos gobiernos desde el 2003, esta política tampoco ha cambiado de forma sustancial, y las iniciativas de refuerzos han pasado a ser lideradas mayormente por instituciones públicas o semipúblicas autonómicas o locales. En estos años se ha avanzado a nivel discursivo sobre la necesidad de promover unos macro-clusters al nivel de plataformas sectoriales y tecnológicas, pero a fecha del 2010 no ha habido actuaciones concretas dignas de destacar. Ver Ahedo (2004).

5 "Innovation efficiency can be as the ratio of turnover from products new to the enterprise and new to the market as a \% of total turnover. It is based on the Community innovation survey and covers at least all enterprises with 10 or more employees. An innovation is a new or significantly improved product (good or service) introduced to the market or the introduction within an enterprise

Aceptado: 12 de mayo de 2011 
of a new or significantly improved process" (Hollanders y Esser, 2007: p. 10).

6 "R\&D is not the only method of innovating. Other methods include technology adoption, incremental changes, imitation, and combining existing knowledge in new ways. With the possible exception of technology adoption, all of these methods require creative effort on the part of the firm's employees and consequently will develop the firm's in-house innovative capabilities. These capabilities are likely to lead to productivity improvements, improved competitiveness, and to new or improved products and processes that could be adopted by other firms. For these reasons, the activities of firms that innovate without performing $R \& D$ are of interest to policy. In general, non-R\&D innovators have lower innovative capabilities than R\&D performing firms, with fewer non-R\&D innovators capable of developing innovations in-house and a smaller percent reporting training or skill upgrading linked to innovation. However, a striking result is that these differences are minor: $71 \%$ of non-R\&D innovators report developing either product or process innovations in-house (compared to 91\% of R\&D performers), $54 \%$ of staff time on innovation is for developing product and process innovations inhouse (compared to 63\% for R\&D performers) and 70\% report training or skills upgrading for innovation (compared to $79 \%$ of R\&D performers)" (Arundel et al., 2008: p. 3).

7 "The results show that a majority of non-R\&D innovators invest in creative innovative activities. Many of these firms should therefore be able to benefit from policy support for their innovative activities. However, policy appears to fail this group of 'neglected' innovators. Only 33\% of non-R\&D innovators report using at least one of six types of innovation support programmes, that do not require $R \& D$ compared to $47 \%$ of $R \& D$ performers. These differences hold after controlling for the innovative capabilities of non-R\&D and $R \& D$ innovators. In particular, firms that innovate primarily through customizing or modifying products or processes are significantly less likely than firms that develop innovations in-house to apply for or use innovation support programmes" (Arundel et al., 2008: p. 4).

8 La lógica privada es fuerte en las comarcas de la Cataluña vieja, como Osona, Ripollés, etc., en donde los principales agentes de innovación tecnológica son de carácter privado: la Universitat de Vic, en la comarca de Osona, y la Fundación Eduard Soler, en el Ripollés. Estos dos agentes han colaborado para implantar en el 2009 uno de los primeros grados universitarios en ingeniería mecatrónica en toda España. Visto el bajo nivel de dinamismo en el sector local de los últimos años, van surgiendo dudas sobre los posibles resultados finales de todos estos esfuerzos locales en estos sectores tecnológicos.

\section{REFERENCIAS}

Ahedo, M. (2004), "Las Asociaciones-Cluster de la Comunidad Autónoma del País Vasco (1986-2002): tradición, interacción y aprendizaje en la colaboración Gobierno-Industria", Ekonomiaz, 53, 114-138.

Ahedo, M. (2006) "Business systems and cluster policies in Basque Country and Catalonia (1991-2003)", European Urban and Regional Studies, 13.1, 5-19.

Arundel, A.; Bordoy, C. y Kanerva, M. (2008), Neglected innovators: How do innovative firms that do not perform R\&D innovate? Results of an analysis of the Innobarometer 2007 Survey, INNO-Metrics Thematic Paper, No. 215.

Asheim, B, y Coenen, L. (2007), "Face-toFace, Buzz and Knowledge- Bases: Socio-spatial implications for learning and innovation policy", Environment and Planning C: Government and Policy, 25: 655-670.

Asheim, B. (2009), "La política regional de innovación de la próxima generación. Cómo combinar los enfoques del impulso de la ciencia y por el usuario en los sistemas regionales de innovación", Ekonomiaz, 70: 86-105.

Barge-Gila, A.; Santamaría, L. y Modrego, A. (2009), "Universities and Technology Institutes. New empirical lessons and perspectives", Working Papers 2009-3, Innova, Catedra Innovación Empresarial-URV.

Biggs, M. (2009), "Self-fulfilling Prophecies, chapter 13", en P. Bearman y Peter Hedström (eds.), The Oxford Handbook of Analytical Sociology, Oxford University Press.

Buesa, M.; Heijs, J.; Martínez Pellitero, M. y Baumert, T. (2006), "Regional systems of innovation and the knowledge production function: the Spanish case", Technovation, 26, 463-472.

Busom, I. (coord.) (2006), La situació de la innovació a Catalunya, Barcelona, Col-lecció d'estudis CIDEM.

Callon, M. (2007), "What does it mean to say that Economics is Performative?", en D. MacKenzie, F. Muniesa y L. Siu (eds.), Do Economists Make Markets?, Princeton University Press, 311-357. 
Casper, S. y Van Waarden, F. (eds.) (2005), Innovation and Institutions: A Multidisciplinary Review of the Study of Innovation Systems, London: Edward Elgar.

Castro, L.; Fernández Mellizo-Soto, M. y Sanz Menéndez, L. (2003), "La importancia de los intereses académicos en la política científica y tecnológica catalana", Papers, 70: 11-40.

Cooke, P. (coordinador) (2006), Constructing regional advantage, EU Commission.

Cooke, P. y Morgan, K. (1998), The Associational Economy, Firms, regions and innovations, Oxford University Press.

Cooke, P.; Heidenreich, M. y Braczyk, H.-J. (eds.) $(1998,2004)$, Regional Innovation Systems. The role of governance in a globalized world, 2nd edition, London, Routledge.

Edquist, C. (1997), Systems of Innovation: Technologies, Institutions and Organisations, London, Pinter.

Fageberg, J.; Mowery, D. C. y Nelson, R. R. (eds.), The Oxford Handbook of Innovation, Oxford University Press.

Hollanders, H. y Esser, F. C. (2007), Measuring innovation efficiency, INNOMetrics Thematic Paper.
Laranja, M.; Uyarra, E. y Flanagan, K. (2008), "Policies for science, technology and innovation: Translating rationales into regional policies in a multi-level setting", Research Policy, 37, 5: 823-835.

Law, J. y Urry, J. (2004), "Enacting the social", Economy and Society, 33: 390410.

Lundvall, B.-Å. (ed.) (1992), National Systems of Innovation: Towards a theory of innovation and interactive learning, London, Pinter.

Lundvall, B.-Å.; Johnson, B.; Andersen, E. S. y Dalum, B. (2002), "National systems of production, innovation and competence building", Research Policy, 31: 213-231.

Maskel, P. y Malmberg, A. (1997), "Localised learning and industrial competitiveness", Cambridge Journal of Economics, 23: 167-185.

Navarro, M. (2009), "Los sistemas regionales de innovación. Una revisión crítica", Ekonomiaz, 70; 25-59.

Uyarra, E. y Flanagan, K. (2009), "La relevancia del concepto de 'sistema regional de innovación' para la formulación de la política de innovación", Ekonomiaz, 70, 150-169.
Olazaran, M. y Gómez-Uranga, M. (2001), Sistemas regionales de innovación, Zarautz, Ed. Universidad del País Vasco.

Olazaran, M. y Otero, B. (2009): "La perspectiva del Sistema Nacional y Regional de Innovación. Balance y recepción en España", Arbor: Ciencia, Pensamiento y Cultura (julio-agosto): 767-779.

Sánz-Menéndez, L. y Castro, L. (2005), "Explicando las políticas de ciencia y tecnología de los gobiernos regionales", Working paper 05-10, Unidad de Políticas Comparadas del CSIC.

Smith, H. L. (2007), "Universities, innovation and territorial development: a review of the evidence", Environment and Planning C: Government and Poli$c y, 25:$ 98-114.

Solà, J.; Sáez, X. y Termes, M. (2006), La innovació $i$ I'R+D industrial a Catalunya, 23 Papers d'Economia Industrial, Barcelona: Generalitat de Catalunya.

Whitley, R. (1999), Divergent Capitalisms. The structuring of changing business systems, Oxford, Oxford University Press.

Whitley, R. (2002), "Developing innovative competences: the role of institutional frameworks", Industrial and Corporate Change, 11 (3): 497-528. 